\title{
Integration of streaming services and TCP data transmission in the Internet
}

\author{
N. Antunes ${ }^{a}$ C. Fricker ${ }^{a}$ F. Guillemin ${ }^{b}$ Ph. Robert ${ }^{a}$ \\ a INRIA, RAP project, Domaine de Voluceau, 78153 Le Chesnay, France \\ ${ }^{\mathrm{b}}$ France Telecom, Division RED, 22300 Lannion, France
}

\begin{abstract}
We study in this paper the integration of elastic and streaming traffic on a same link in an IP network. We are specifically interested in the computation of the mean bit rate obtained by a data transfer. For this purpose, we consider that the bit rate offered by streaming traffic is low, of the order of magnitude of a small parameter $\varepsilon \ll 1$ and related to an auxiliary stationary Markovian process $(X(t))$. Under the assumption that data transfers are exponentially distributed, arrive according to a Poisson process, and share the available bandwidth according to the ideal processor sharing discipline, we derive the mean bit rate of a data transfer as a power series expansion in $\varepsilon$. Since the system can be described by means of an $M / M / 1$ queue with a time-varying server rate, which depends upon the parameter $\varepsilon$ and process $(X(t))$, the key issue is to compute an expansion of the area swept under the occupation process of this queue in a busy period. We obtain closed formulas for the power series expansion in $\varepsilon$ of the mean bit rate, which allow us to verify the validity of the so-called reduced service rate at the first order. The second order term yields more insight into the negative impact of the variability of streaming flows.
\end{abstract}

Key words: variable $M / M / 1$ queue, perturbation theory, processor-sharing

\section{Introduction}

The emergence of the Internet as the universal multi-service network raises major traffic engineering problems, in particular with regard to the coexistence on the same transmission links of real time and data services. As a matter of fact, these two types of services have different requirements in terms of transfer delay and loss, data transmission being very sensitive to packet loss but relatively tolerant to delay whereas real time services have strict transfer delay constraints. While classical data transfers are usually controlled by TCP (Transmission Control Protocol), which aims at achieving a fair bandwidth 
allocation at a bottleneck link (see Massoulié and Roberts (1) for a discussion on modeling TCP at the flow level and processor sharing), real time services most of the time are supported by the unreliable UDP protocol, even if some transmission control can be performed by upper layers (e.g., RTCP). Real time services thus reduce the transmission capacity for data transfers.

This problem has been addressed by Delcoigne et al. (2), where stochastic bounds have been obtained for the bit rate seen by a TCP data transfer, when elastic traffic and unresponsive streaming flows are multiplexed on a same link (see also Bonald and Proutière (3))). From a theoretical point of view, this problem can be seen as the analysis of a priority system, where streaming flows have priority over data traffic. In this context, a usual approximation (referred to as Reduced Service Rate, RSR) consists of assuming that everything happens as if the service rate for data were reduced by the mean bit rate offered by streaming flows. This approximation has been investigated for the number of active data flows by Antunes et al. (四), when the load offered by streaming flows is very small. We note that the same kind of problem has been addressed in the technical literature by Núñez-Queija and Boxma (5) in the context of ABR service in ATM networks and more recently by Núñez-Queija (6; 7) via matrix analysis for systems described by means of quasi birth and death processes. In a similar context, Núñez-Queija et al. (8) use a perturbation technique for studying a priority system, where priority traffic offers a small load. Although the systems considered in this paper are quite general, no explicit expressions for the terms of the expansions are provided. Finally, note that systems with different speeds are also of interest for analyzing the coexistence of different traffic types (9).

In this paper, we investigate the mean bit rate obtained by a data transfer when elastic traffic and unresponsive streaming flows are multiplexed on a same transmission link. Along the same line of investigations as Antunes et al. (四), because of the real difficulty of the problems, the mean load offered by streaming flows is supposed to be very small (controlled by a parameter $\varepsilon \ll 1$ ) and a perturbation analysis for the analysis of the mean bit rate is done. It is assumed that elastic flows arrive according to a Poisson process and share the available bandwidth according to the processor sharing discipline. In addition, to simplify the computations, we assume that the service time required by data transfers is exponentially distributed with parameter $\mu$. Thus, we have to deal with an $M / M / 1$ queue with a time-varying server rate, which depends upon the instantaneous number of active streaming flows. The exponential distribution of the service time is of course not realistic in practice, but as it will be seen later, the mathematical analysis is already very difficult in the case so that our work should be considered as a first step in this domain.

To compute the mean bit rate of a data transfer, we consider the quantity $\mathcal{A}=\int_{0}^{B} L(s) d s$, where $B$ is the length of the busy period and $L(t)$ is the 
number of customers at time $t$ in the $M / M / 1$ queue under consideration. The quantity $\mathcal{A}$ is equal to the cumulative waiting time in the $M / M / 1$ queue and also represents the amount of data served during a busy period. In the case of the $M / M / 1$ PS sharing queue, if $\mathbb{E}(d)$ represents the mean bit rate obtained by a data transfer, we have $\mathbb{E}(\mathcal{A})=\mathbb{E}(N) \mathbb{E}(S) / \mathbb{E}(d)=\mathbb{E}(B) / \mathbb{E}(d)$, where $\mathbb{E}(S)$ is the mean service time (equal to $1 / \mu$ ) and $\mathbb{E}(N)$ is the mean number of customers served in a busy period. Thus, the computation of $\mathbb{E}(\mathcal{A})$ allows us to estimate $\mathbb{E}(d)$, since the quantity $\mathbb{E}(B)$ has been computed by Antunes et al. (4) as a power series expansion of $\varepsilon$. Note that in the case of a classical $M / M / 1$ queue, we have $E(B)=1 /(\mu(1-\rho))$ and $\mathbb{E}(\mathcal{A})=1 /\left(\mu(1-\rho)^{2}\right)$, which yields $\mathbb{E}(d)=(1-\rho)$, where $\rho$ is the offered load. This is the classical result for an $M / G / 1$ PS queue, which states that the mean bit rate obtained by a data transfer is $(1-\rho)$ times the server rate (taken as unity in this paper); see Massoulié and Roberts (11).

In this paper, we derive a power series expansion in $\varepsilon$ of the quantity $\mathbb{E}(\mathcal{A})$ in the case of an $M / M / 1$ queue, whose server rate is modulated by an auxiliary process $(X(t))$. We specifically assume that the server rate at time $t$ is $\mu+$ $\varepsilon p(X(t))$ for some function $p$ satisfying regularity assumptions, the process $(X(t))$ being stationary, ergodic, and Markovian. The objective of this paper is, first to check the validity of the RSR approximation, which claims that everything happens as if the server rate were frozen at the value $\mu+\varepsilon \mathbb{E}[p(X(0))]$ and, second, to get some qualitative insight on the impact of the variability of streaming flows on elastic traffic.

The organization of this paper is as follows: The model is described in Section 2 , where the main result concerns the power series expansion in $\varepsilon$ of $\mathbb{E}(\mathcal{A})$, which is the key quantity for computing the mean bit rate of a data transfer. In Section 3, the main result is applied to obtain the expansion of the mean bit rate of a data transfer and to analyze different special cases. Some concluding remarks are presented in Section 4. The quite technical proof of the main result is sketched in the Appendix.

\section{Model description}

Throughout this paper we consider a stable $M / M / 1$ queue with arrival rate $\lambda$ and service rate $\mu$; the load $\rho=\lambda / \mu<1$. Let $L(t)$ denote the number of customers at time $t$. The invariant distribution $\pi$ of $(L(t))$ is geometrically distributed with parameter $\rho$.

Let $B$ denote the duration of a busy period starting with one customer, that is, $B=\inf \{s \geq 0: L(s)=0\}$, given $L(0)=1$. For $x \geq 1$, let $B_{x}$ denote the duration of a busy period starting with $x$ customers. Note that $B_{1} \stackrel{\text { dist. }}{=} B$. In 
the following, when the variables $B, B_{1}$ and $B_{1}^{\prime}$ are used in the same expression, they are assumed to be independent with the same distribution as $B$.

The quantity $\mathcal{A}$ defined in the Introduction represents the area swept under the occupation process in a busy period. When several busy cycles are considered, the notation $\mathcal{A}_{B}$ will be used to indicate that the area is calculated for the corresponding busy period of length $B$. By definition, the relation $\mathcal{A} \geq B$ holds, the excess will be denoted by $\overline{\mathcal{A}} \stackrel{\text { def. }}{=} \overline{\mathcal{A}}_{B} \stackrel{\text { def. }}{=} \mathcal{A}_{B}-B$. This queue will be referred to as the standard queue denoted, for short, by S-Queue.

Streaming flows impact data transfers by reducing the amount of available bandwidth. This situation is described by introducing an $M / M / 1$ queue with arrival rate $\lambda$ and varying service rate driven by an ergodic Markov process $(X(t))$ taking values in a state space $\mathcal{S}$. Typically, the state space of the environment is a finite, countable set when $(X(t))$ is a Markov Modulated Poisson Process or $\mathcal{S}=\mathbb{R}$ in the case of a diffusion, for instance an Ornstein-Uhlenbeck process (see Fricker et al. (10)). The invariant measure of the process $(X(t))$ is denoted by $\nu$. The Markovian notation $\mathbb{E}_{x}(\cdot)$ will refer only to the initial state $x$ of the Markov process $(X(t))$.

Let $\widetilde{L}^{\varepsilon}(t)$ be the number of customers of the queue at time $t$. The process $\left(\widetilde{L}^{\varepsilon}(t), X(t)\right)$ is a Markov process. If $X(t)=x$ and $L(t)=n>0$, then the service rate is given by $\mu+\varepsilon p(x)$ for some function $p(x)$ on the state space of the environment $\mathcal{S}$ and some small parameter $\varepsilon \geq 0$. For $t \geq 0$, let us define the quantities $p^{+}(t)=\max (p(t), 0)$ and $p^{-}(t)=\max (-p(t), 0)$ so that $p(t)=p^{+}(t)-p^{-}(t)$. At time $t$, the additional capacity is therefore $\varepsilon p^{+}(X(t))$ and $\varepsilon p^{-}(X(t))$ is the capacity lost.

In the rest of this paper, we make the two following assumptions:

the function $p(x)$ is bounded $\left(H_{1}\right)$ and $\lambda+\varepsilon \sup (|p(x)|: x \in \mathcal{S})<\mu\left(H_{2}\right)$. If assumption $\left(H_{2}\right)$ holds (it is a suffifient but not necessary condition) then the queue is stable and, in this case, the duration $\widetilde{B}^{\varepsilon}$ of a busy period starting with one customer, $\widetilde{B}^{\varepsilon}=\inf \left\{s \geq 0: \widetilde{L}^{\varepsilon}(s)=0 \mid \widetilde{L}^{\varepsilon}(0)=1\right\}$, is a.s. finite. The queue with time-varying service rate as defined above will be referred to as the perturbated queue, denoted, for short, by P-Queue. The case $\varepsilon=0$ obviously corresponds to the S-Queue. The area for the perturbated queue over a busy cycle is defined as

$$
\widetilde{\mathcal{A}}^{\varepsilon}=\int_{0}^{\widetilde{B}^{\varepsilon}} \widetilde{L}^{\varepsilon}(s) d s
$$

The basic idea of the perturbation analysis carried out in this paper for the quantity $\widetilde{\mathcal{A}}^{\varepsilon}$ defined by the above equation is to construct a coupling between the busy periods of the processes $\left(\widetilde{L}^{\varepsilon}(t)\right)$ and $(L(t))$. Provided that for both queues the arrival process is the same Poisson process with parameter $\lambda$, we add and remove departures as follows. 
Additional departures. When $p^{+}(X(t))>0$, there is additional capacity when compared with the S-Queue and more departures can take place. These additional departures are counted by means of a point Process $\mathcal{N}^{+}=$ $\left(t_{i}^{+}\right)$, with $0<t_{1}^{+} \leq t_{2}^{+} \leq \cdots$, which is a non-homogeneous Poisson process on $\mathbb{R}_{+}$with intensity given by $t \rightarrow \varepsilon p^{+}(X(t))$. Conditionally on $(X(t))$, the number of points of $\mathcal{N}^{+}$in the interval $[a, b]$ is Poisson with parameter $\varepsilon \int_{a}^{b} p^{+}(X(s)) d s$. In particular the distribution of the location $t_{1}^{+} \geq 0$ of the first point of $\mathcal{N}^{+}$after 0 is given, for $x \geq 0$, by

$$
\mathbb{P}\left(t_{1}^{+} \geq x\right)=\mathbb{P}\left(\mathcal{N}^{+}([0, x])=0\right)=\mathbb{E}\left[\exp \left(-\varepsilon \int_{0}^{x} p^{+}(X(s)) d s\right)\right] .
$$

Removing Departures On the other hand, when $p^{-}(X(t))>0$, the server rate is smaller than in the S-queue. Let $\mathcal{N}_{\mu}=\left(t_{i}\right)$, a Poisson process with intensity $\mu$ on $\mathbb{R}_{+}$which represents the non-decreasing sequence of instants when the customer of the S-queue (if not empty) may leave the queue. We denote by $\mathcal{N}^{-}$the point process obtained as follows: For $s>0$, a point at $s$ of the Poisson process $\mathcal{N}_{\mu}$ is a point of $\mathcal{N}^{-}$with probability $\varepsilon p^{-}(X(s)) / \mu$. (Note that this number is $\leq 1$ by assumption $\left(H_{2}\right)$.) The point process $\mathcal{N}^{-}$ is Poisson with intensity $s \rightarrow \varepsilon p^{-}(X(s))$. A point of $\mathcal{N}^{-}$is called a marked departure. The points of $\mathcal{N}^{-}$are denoted by $0<t_{1}^{-} \leq t_{2}^{-} \leq \cdots \leq t_{n}^{-} \leq \cdots$. By definition,

$$
\mathbb{P}\left(t_{1}^{-} \geq x\right)=\mathbb{E}\left(\prod_{t_{i}, t_{i} \leq x}\left(1-\frac{\varepsilon p^{-}\left(X\left(t_{i}\right)\right)}{\mu}\right)\right), \quad x \geq 0 .
$$

The processes defined above are non homogeneous Poisson processes (see Grandell (11) for an account on this topic). The main result of this paper is the expansion in power series of $\varepsilon$ up to the second order of $\mathbb{E}\left(\widetilde{\mathcal{A}}^{\varepsilon}\right)$.

Theorem 1 The second order expansion of the area swept under the occupation process of the perturbated queue during a busy period is given by

$$
\mathbb{E}\left(\widetilde{\mathcal{A}}^{\varepsilon}\right)=\mathbb{E}(\mathcal{A})-\varepsilon \frac{\mathbb{E}[p(X(0))](\lambda+\mu)}{(\mu-\lambda)^{3}}-\varepsilon^{2}\left(a_{+}+a_{-}+a_{ \pm}\right)+o\left(\varepsilon^{2}\right),
$$

where the coefficients $a_{+}, a_{-}, a_{ \pm}$are defined below by Equations (A.4), (A.5), and (A.6), respectively.

\section{Applications}

In this section, as an application of Theorem 1, we evaluate the mean bit rate $\mathbb{E}\left(d_{\varepsilon}\right)$ obtained by an elastic data transfer when the server rate is perturbated by the presence of streaming flows (coming through the term $\varepsilon p(X(t))$ in the 
service rate of the perturbated $M / M / 1$ queue, equal to $\mu+\varepsilon p(X(t)))$. As mentioned in the Introduction, we have $\mathbb{E}\left(\widetilde{\mathcal{A}}^{\varepsilon}\right)=\mathbb{E}\left(\widetilde{B}^{\varepsilon}\right) / \mathbb{E}\left(d_{\varepsilon}\right)$. The average of the duration $\widetilde{B}^{\varepsilon}$ of the corresponding busy period has been studied by Antunes et al. (4) and can be expanded in power series of $\varepsilon$ as follows.

Theorem 2 The expansion of $\mathbb{E}\left(\widetilde{B}^{\varepsilon}\right)$, the mean duration of a busy period, is given by $\mathbb{E}\left(\widetilde{B}^{\varepsilon}\right)=1 /(\mu-\lambda)-\varepsilon \mathbb{E}_{\nu}(p(X(0))) /(\mu-\lambda)^{2}+\left(b_{-}-b_{+}\right) \varepsilon^{2}+o\left(\varepsilon^{2}\right)$. where $b_{+}$and $b_{-}$are given by, with the notation of Proposition 8 ,

$$
\begin{gathered}
b_{+}=-\frac{1}{\mu} \mathbb{E}\left(\int_{0}^{B}(B-v) \mathbb{E}_{\nu}\left(p^{+}(X(0)) p^{+}(X(v))\right) d v\right) \\
-\frac{1}{\mu^{2}(1-\rho)} \mathbb{E}\left(\sum_{i=1}^{H} \sum_{j=1}^{N_{i}} \int_{0}^{A_{i}} p^{+}(X(u)) p^{-}\left(X\left(D_{i}^{j}\right)\right) d u\right), \\
b_{-}=\frac{1}{\mu^{2}(1-\rho)}\left(-\mathbb{E}\left(\sum_{i=1}^{N} \int_{0}^{B+B_{1}} p^{-}\left(X\left(D_{i}\right)\right) p^{+}(X(s)) d s\right)\right. \\
\left.+\frac{1}{\mu} \mathbb{E}\left(\sum_{i=1}^{N} \sum_{k=1}^{N^{\prime}} p^{-}\left(X\left(D_{i}\right)\right) p^{-}\left(X\left(B+D_{k}^{\prime}\right)\right)\right)\right) .
\end{gathered}
$$

By using Equations (4) and (5) and Theorem 1, straightforward computations show that the quantity $\mathbb{E}\left(d_{\varepsilon}\right)$ can then expanded in power series of $\varepsilon$ as follows.

Proposition 1 The mean bit rate of an elastic data transfer can be expanded in power series of $\varepsilon$ as

$$
\mathbb{E}\left(d_{\varepsilon}\right)=1-\rho+\frac{\rho \mathbb{E}_{\nu}(p(X(0)))}{\mu} \varepsilon+c \varepsilon^{2}+o\left(\varepsilon^{2}\right),
$$

where the coefficient $c$ is given by

$$
c=\mathbb{E}_{\nu}(p(X(0)))^{2} \frac{\rho(1+\rho)}{\mu^{2}(1-\rho)}+\mu(1-\rho)^{2}\left((1-\rho)\left(a_{+}+a_{-}+a_{ \pm}\right)+b_{-}-b_{+}\right),
$$

the quantities $a_{+}, a_{-}, a_{ \pm}, b_{+}$and $b_{-}$being defined by equations (A.4), (A.5), (A.6), (4) and (5), respectively.

From Equation (6), we immediately deduce that as far as the first order term is concerned, the RSR approximation is valid: for an $M / M / 1$ queue with service rate $\mu+\varepsilon \mathbb{E}_{\nu}(p(X(0)))$, the mean bit rate denoted by $\hat{d}$ obtained by a customer is given by $\mathbb{E}(\hat{d})=1-\lambda /\left(\mu+\varepsilon \mathbb{E}_{\nu}[p(X(0))]\right)=1-\rho+\varepsilon \rho \mathbb{E}_{\nu}[p(X(0))] / \mu+o(\varepsilon)$ Unfortunately, the coefficient $c$ defined by Equation (7) intricately depends upon the correlation structure of the modulating process $(X(t))$ and the dynamics of the $M / M / 1$ queue. Because of this complexity, three cases of practical interest in the following are considered: non-positive perturbation func- 
tions, non-negative perturbation functions, and special environments (namely, fast and slow environments).

\subsection{Non-positive Perturbation Functions}

We assume in this section that the perturbation function is non-positive so that the environment uses a part of the capacity of the $M / M / 1$ queue with constant service rate $\mu$. This application is motivated by the following practical situation. Coming back to the coexistence of elastic and streaming traffic in the Internet, assume that priority is given to streaming traffic in a buffer of a router. The bandwidth available for non-priority traffic is the transmission link reduced by the rate of streaming traffic. Denoting by $\operatorname{\varepsilon r}(X(t))$ the rate of streaming traffic at time $t$ (for instance $\varepsilon$ may represent the peak rate of a streaming flow and $r(X(t))$ the number of such flows active at time $t)$, the service rate available for non-priority traffic is $\mu-\varepsilon r(X(t))$. Setting $p(x)=-r(x)$, the function $p(x)$ is non-positive.

Proposition 2 When $p^{+} \equiv 0$, if $\hat{d}$ is the mean bit rate of the $M / M / 1$ queue with service rate $\mu+\varepsilon \mathbb{E}[p(X(0))]$, then with the notation of Proposition 8,

$$
\begin{aligned}
\lim _{\varepsilon \rightarrow 0} \frac{1}{\varepsilon^{2}} \mathbb{E}\left(d_{\varepsilon}-\hat{d}\right)= & -\frac{(1-\rho)^{2}}{\mu^{2}} \mathbb{E}\left(\sum_{1 \leq i<j \leq N} C_{p}\left(X\left(D_{j}-D_{i}\right)\right)\right) \\
& -\frac{(1-\rho)^{3}}{\mu} \mathbb{E}\left(\sum_{i=1}^{N} \sum_{j=1}^{N^{\prime}} C_{p}\left(B-D_{i}+D_{j}^{\prime}\right)\left(B_{1}-D_{j}^{\prime}\right)\right),
\end{aligned}
$$

where the function $C_{p}(u)$ is defined for $u \geq 0$ by

$$
C_{p}(u)=\mathbb{E}_{\nu}[p(X(0)) p(X(u))]-\mathbb{E}_{\nu}[p(X(0))]^{2}
$$

and is, up to the factor $\varepsilon^{2}$, the auto-covariance function of the variable capacity of the perturbated queue.

The above result shows that if the process $(X(t))$ is positively correlated, i.e. $C_{p}(\cdot) \geq 0$, then $\lim _{\varepsilon \rightarrow 0} \mathbb{E}\left(d_{\varepsilon}-\hat{d}\right) / \varepsilon^{2}<0$. The environment has therefore a negative impact on the performances of the system in this case.

Proof. The terms $a_{+}, a_{ \pm}$and $b_{+}$in the coefficient $c$ defined by Equation (7) are equal to 0 . In addition, one easily checks that

$$
\begin{aligned}
(1-\rho) a_{-}+b_{-} & =-\frac{1}{\mu^{3}} \mathbb{E}\left(\sum_{1 \leq i<j \leq N} p^{-}\left(X\left(D_{i}\right)\right) p^{-}\left(X\left(D_{j}\right)\right)\right) \\
& -\frac{(1-\rho)}{\mu^{2}} \mathbb{E}\left(\sum_{i=1}^{N} \sum_{k=1}^{N^{\prime}} p^{-}\left(X\left(D_{i}\right)\right) p^{-}\left(X\left(B+D_{k}^{\prime}\right)\right)\left(B_{1}-D_{j}^{\prime}\right)\right) .
\end{aligned}
$$

One concludes by using Expansion (6). 


\subsection{Non-negative Perturbation Functions}

It is assumed in this section that $p^{-} \equiv 0$. We have the following result, which is the analogue of Proposition 2 for this case. Contrary to the above proposition, the expansion has a more explicit expression. Its (straightforward) proof is omitted.

Proposition 3 When $p^{-} \equiv 0$, with the same notations as in Proposition 2.

$$
\begin{aligned}
\lim _{\varepsilon \rightarrow 0} \frac{1}{\varepsilon^{2}} \mathbb{E}\left(d_{\varepsilon}-\hat{d}\right)=(1-\rho)^{3}( & \mathbb{E}\left(\int_{0}^{B}(B-v) C_{p}(v) d v\right) \\
& \left.-\frac{(1-\rho) \mu}{2} \mathbb{E}\left(\int_{0}^{B}(B-v)^{2} C_{p}(v) d v\right)\right) .
\end{aligned}
$$

An integration by parts and some calculations give the following corollary.

Corollary 3 When the correlation function of the environment is exponentially decreasing, i.e., when $C_{p}(x)=\operatorname{Var}[p(X(0))] e^{-\alpha x}$ for all $x \geq 0$ and some $\alpha>0$, then

$$
\lim _{\varepsilon \rightarrow 0} \frac{1}{\varepsilon^{2}} \mathbb{E}\left(d_{\varepsilon}-\hat{d}\right)=\frac{1}{\mu^{2}}\left(\mathbb{E}\left(e^{-\alpha B^{* *}}\right)-\frac{1+\rho}{1-\rho} \mathbb{E}\left(e^{-\alpha B^{* * *}}\right)\right)
$$

with the convention that, if $Z$ is some integrable non-negative random variable, the density on $\mathbb{R}_{+}$of the variable $Z^{*}$ is defined as

$$
\mathbb{P}\left(Z^{*} \geq x\right)=\mathbb{P}(Z \geq x) / \mathbb{E}(Z)
$$

for $x \geq 0$, and $Z^{* *}$ stands for $\left(Z^{*}\right)^{*}$, and $Z^{* *}$ for $\left(Z^{* *}\right)^{*}$.

When $\alpha$ is small, the right hand side of Equation (11) is equivalent to the quantity $-2 \rho / \mu^{2}<0$. This shows that non-negative perturbation functions have a negative impact at the second order on the mean bit rate of elastic data transfers.

Remark. This result could maybe be extended to a perturbated function with a non-constant sign but it is out of reach because of the complexity of the secund order term.

\subsection{Fast and slow Environments}

The performance of the system in two limit regimes, called fast and slow environments, are now evaluated. These regimes are very useful, since performance in the limit regimes is insensitive and only depends on appropriately defined parameters. Such a situation has also been analyzed by Delcoigne et al. (2) through stochastic bounds. 
The environment is scaled by a factor $\alpha>0$, such that at time $t$ the environment is supposed to be $X(\alpha t)$. The behavior when $\alpha$ goes to infinity and zero is investigated.

When the parameter $\alpha$ is very large, the environment process approximately averages the capacity of the variable queue. For a large $\alpha$ and for $t$ and $h>0$, the total service capacity available during $t$ and $t+h$ is given by

$$
\mu h+\varepsilon \int_{t}^{t+h} p(X(\alpha u)) d u \stackrel{\text { dist. }}{=} \mu h+\varepsilon \frac{1}{\alpha} \int_{0}^{\alpha h} p(X(u)) d u \sim(\mu+\varepsilon \mathbb{E}[p(X(0))]) h
$$

using the stationarity of $(X(t))$ and the ergodic theorem. Thus, when $\alpha$ tends to infinity, the variations completely vanish and the service rate reduces to a constant.

On the other hand, for small values of $\alpha$, the environment process remains almost constant over the busy period of the P-Queue. As $\alpha$ goes to 0 , the variation disappears and the environment is frozen in the initial state of the process: the service rate is constant and equal to $\mu+\varepsilon p(X(0))$.

This intuitive picture is rigorously established in the next proposition. In the following a general perturbation function $p$ is considered together with some stationary Markov process $(X(t))$ with invariant probability distribution $\nu$. It is assumed that it verifies a mixing condition such as

$$
\lim _{t \rightarrow+\infty} \mid \mathbb{E}\left[f(X(0) g(X(t))]-\mathbb{E}_{\nu}(f) \mathbb{E}_{\nu}(g) \mid=0\right.
$$

for any Borelian bounded functions $f$ and $g$ on the state space $\mathcal{S}$. Note that this condition is not restrictive in general since it is true for any ergodic Markov process with a countable (or finite) state space or for any diffusion on $\mathbb{R}^{d}$.

Under the above assumptions, we have the following result; the proof relies on the use of the mixing condition (12) and can be found in the paper by Antunes et al. (12).

Proposition 4 When the environment is given by $(X(\alpha t))$ and Relation (12) holds, then when $\varepsilon$ tends to $0, \mathbb{E}\left(d_{\varepsilon}\right)-\mathbb{E}(\hat{d})=\Psi(\alpha) \varepsilon^{2}+o\left(\varepsilon^{2}\right)$ where

$$
\lim _{\alpha \rightarrow+\infty} \Psi(\alpha)=-\rho \frac{\mathbb{E}_{\nu}(p(X(0)))^{2}}{\mu^{2}} \text { and } \lim _{\alpha \rightarrow 0} \Psi(\alpha)=-\rho \frac{\mathbb{E}_{\nu}\left(p(X(0))^{2}\right)}{\mu^{2}}
$$

The fast and slow environment provide an explicit estimate of the second order term, where the slow environment yields a worst performance of the perturbated queue. It is not clear that these limit regimes give a lower and upper bound of the performance of the queue. 


\section{Conclusion}

We have investigated in this paper the impact on the performance of elastic data transfers of the presence of streaming flows, when both kinds of traffic are multiplexed on a same link of an IP network. By assuming that the perturbation due to streaming flows is of small magnitude, a perturbation analysis can be performed in order to obtain explicit results for the mean bit rate achieved by a data transfer, under the assumption that elastic streams share the available bandwidth according to the processor sharing discipline. It turns out that at the first order, the so-called RSR approximation is valid. This is not the case for the second order, for which the variability of streaming flows seem to have a negative impact, at least for the three cases examined here.

Further investigations are needed in order to estimate the degradation suffered by data transfers at the second order. The perturbation analysis carried out in this paper is possible, because we have assumed that streaming flows offer a very small contribution to the total load. When this is not the case, new tools have to be developed to estimate the quality of data transfers.

\section{A Appendix: Proof of Theorem 1}

Since the derivation of the expansion of the average area is somewhat technical, we begin with the simplest case, which is the first order expansion. In the following, we set $\Delta^{\varepsilon}=\mathbb{E}(\mathcal{A})-\mathbb{E}\left(\widetilde{\mathcal{A}}^{\varepsilon}\right)$.

\section{A.1 First order term}

We first consider an additional departure. Define $\mathcal{E}_{+}=\left\{t_{1}^{+} \leq B, t_{1}^{-} \geq t_{1}^{+}+\right.$ $\left.B_{L\left(t_{1}^{+}\right)-1}\right\}$, where $B_{L\left(t_{1}^{+}\right)-1}$ is the duration between the $t_{1}^{+}$and the first time when the S-Queue has one customer (see Figure A.1-(b)). On this event, an additional departure is added and the busy period of the P-Queue finishes before a departure is canceled. For the first order term of the expansion of the mean value of $\Delta^{\varepsilon}$ on the event $\mathcal{E}_{+}$, we only need to consider the case where there is only one additional departure during the busy period of the P-Queue. The probability that two additional jumps occur in the same busy period is of the order of magnitude of $\varepsilon^{2}$ since the intensity of the associated Poisson process is proportional to $\varepsilon$. 
Lemma 5 In the case of a single additional departure

$$
\mathbb{E}\left(\Delta^{\varepsilon} \mathbb{1}_{\mathcal{E}_{+}}\right)=\varepsilon \frac{\mathbb{E}_{\nu}\left[p^{+}(X(0))\right](\lambda+\mu)}{(\mu-\lambda)^{3}}+o(\varepsilon)
$$

Proof. The difference $\Delta^{\varepsilon}$ on the event $\left\{t_{1}^{+} \leq B, t_{2}^{+}>t_{1}^{+}+B_{L\left(t_{1}^{+}\right)-1}, t_{1}^{-} \geq\right.$ $\left.t_{1}^{+}+B_{L\left(t_{1}^{+}\right)-1}\right\}$ is the sum of two disjoint areas (see Figure A.1-(a)). The first one is given by the distance between $t_{1}^{+}$and the end of the busy period of the S-Queue. By the strong Markov property at the stopping time $\widetilde{B}^{\varepsilon}$, conditionally on the event $\left\{t_{1}^{+} \leq B, t_{2}^{+} \geq t_{1}^{+}+B_{L\left(t_{1}^{+}\right)-1}, t_{1}^{-} \geq t_{1}^{+}+B_{L\left(t_{1}^{+}\right)-1}\right\}$, the S-Queue starts at time $\widetilde{B}^{\varepsilon}$ an independent busy period with one customer (with duration $B_{1}$ ). The second area of $\Delta^{\varepsilon}$ is then given by the area of the sub-busy periods in $B_{1}$, i.e. periods when $t \rightarrow L(t)$ is $>1$ in the second b.p.
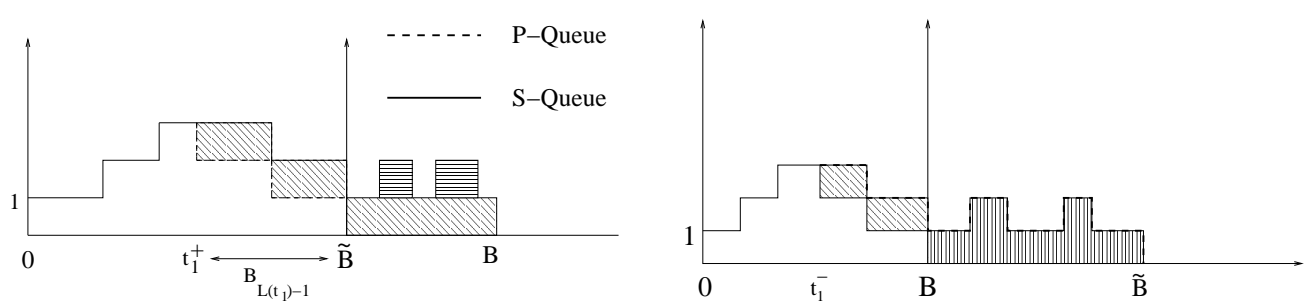

Fig. A.1. (a) One additional departure - (b) One marked departure

It follows that

$$
\begin{gathered}
\mathbb{E}\left(\Delta^{\varepsilon} \mathbb{1}_{\mathcal{E}_{+}}\right)=\mathbb{E}\left(\Delta^{\varepsilon} \mathbb{1}_{\left\{t_{1}^{+} \leq B, t_{2}^{+} \geq t_{1}^{+}+B_{L\left(t_{1}^{+}\right)-1}, t_{1}^{-} \geq t_{1}^{+}+B_{L\left(t_{1}^{+}\right)-1}\right\}}\right)+o(\varepsilon)= \\
\mathbb{E}\left(\left(B-t_{1}^{+}\right) \mathbb{1}_{\left\{t_{1}^{+} \leq B, t_{2}^{+} \geq t_{1}^{+}+B_{L\left(t_{1}^{+}\right)-1}, t_{1}^{-} \geq t_{1}^{+}+B_{L\left(t_{1}^{+}\right)-1}\right\}}\right) \\
+\mathbb{E}\left(\mathcal{A}_{B_{1}}-B_{1}\right) \mathbb{P}\left(t_{1}^{+} \leq B, t_{2}^{+} \geq t_{1}^{+}+B_{L\left(t_{1}^{+}\right)-1}, t_{1}^{-} \geq t_{1}^{+}+B_{L\left(t_{1}^{+}\right)-1}\right) \\
=\mathbb{P}\left(t_{1}^{+} \leq B\right) \mathbb{E}\left(\mathcal{A}_{B_{1}}-B_{1}\right)+\mathbb{E}\left(\left(B-t_{1}^{+}\right) \mathbb{1}_{\left\{t_{1}^{+} \leq B\right\}}\right)+o(\varepsilon) .
\end{gathered}
$$

Equation (1) and the boundedness of $p$ give that

$$
\begin{aligned}
\mathbb{P}\left(t_{1}^{+} \leq B\right) & =1-\mathbb{E}\left[\exp \left(-\varepsilon \int_{0}^{B} p^{+}(X(s)) d s\right)\right] \\
=\varepsilon \mathbb{E}\left[\int_{0}^{B} p^{+}(X(s)) d s\right]+o(\varepsilon)=\varepsilon \mathbb{E}(B) \mathbb{E}\left[p^{+}(X(0))\right]+o(\varepsilon) & =\frac{\varepsilon}{\mu-\lambda} \mathbb{E}\left[p^{+}(X(0))\right]+o(\varepsilon)
\end{aligned}
$$

by independence between $B$ and $(X(t))$ and by the stationarity of $(X(t))$. 
Similarly,

$$
\begin{aligned}
\mathbb{E}\left(\left(B-t_{1}^{+}\right) \mathbb{1}_{\left\{t_{1}^{+} \leq B\right\}}\right) & =\mathbb{E}\left(\int_{0}^{B} \varepsilon p^{+}(X(u)) e^{-\varepsilon \int_{0}^{u} p^{+}(X(s)) d s}(B-u) d u\right) \\
& =\varepsilon \mathbb{E}\left[p^{+}(X(0))\right] \frac{\mathbb{E}\left(B^{2}\right)}{2}+o(\varepsilon)
\end{aligned}
$$

Since $\mathbb{E}\left(B^{2}\right)=2 / \mu^{2}(1-\rho)^{3}$ and $\mathbb{E}\left(\mathcal{A}_{B_{1}}\right)=\mu /(\mu-\lambda)^{2}$ (see for instance standard books such as Cohen (13))). The lemma is proved.

We now turn to the case, when there is one removed departure. On the event $\mathcal{E}_{-}=\left\{t_{1}^{-} \leq B, B+B_{1} \leq t_{1}^{+}\right\}$, a marked departure occurs and no departures are added before the completion of the busy period $B_{1}$. We derive the first order expansion of the mean value of $\Delta^{\varepsilon}$ on $\mathcal{E}_{-}$.

Assume that there is only one marked departure and no additional jumps during the busy period of the P-Queue. In this case, at the end of the busy period of the S-Queue, the P-Queue has one customer and the difference is the distance between $t_{1}^{-}$and the end of the busy period $B$. At time $B$, the P-Queue starts a busy period with one customer and provided that there are no marked and additional departures during $\left(B, B+\widetilde{B}^{\varepsilon}\right)$, the difference has the same distribution as the area of a busy period $B_{1}$ of the standard queue (see Figure A.1-(b)).

Lemma 6 In the case of a single marked departure

$$
\mathbb{E}\left(\Delta^{\varepsilon} \mathbb{1}_{\mathcal{E}_{-}}\right)=-\varepsilon \frac{\mathbb{E}\left[p^{-}(X(0))\right](\lambda+\mu)}{(\mu-\lambda)^{3}}+o(\varepsilon)
$$

Proof. By using the same arguments as before, one obtains the relation

$$
\begin{aligned}
\mathbb{E}\left(\Delta^{\varepsilon} \mathbb{1}_{\mathcal{E}_{-}}\right)= & \mathbb{E}\left(\Delta^{\varepsilon} \mathbb{1}_{\left\{t_{1}^{-} \leq B, t_{2}^{-} \geq B+B_{1}, t_{1}^{+} \geq B+B_{1}\right\}}\right)+o(\varepsilon) \\
& =-\mathbb{E}\left(\left(B-t_{1}^{-}+\mathcal{A}_{B_{1}}\right) \mathbb{1}_{\left\{t_{1}^{-} \leq B, t_{2}^{-} \geq B+B_{1}, t_{1}^{+} \geq B+B_{1}\right\}}\right)+o(\varepsilon)
\end{aligned}
$$

Hence, $\mathbb{E}\left(\Delta^{\varepsilon} \mathbb{1}_{\mathcal{E}_{-}}\right)=-\mathbb{E}\left(\left(B-t_{1}^{-}+\mathcal{A}_{B_{1}}\right) \mathbb{1}_{\left\{t_{1}^{-} \leq B, t_{2}^{-} \geq B+B_{1}, t_{1}^{+} \geq B+B_{1}\right\}}\right)+o(\varepsilon)$, so that $\mathbb{E}\left(\Delta^{\varepsilon} \mathbb{1}_{\mathcal{E}_{-}}\right)=-\mathbb{E}\left(\left(B-t_{1}^{-}\right) \mathbb{1}_{\left\{t_{1}^{-} \leq B\right\}}\right)-\mathbb{E}\left(\mathcal{A}_{B_{1}}\right) \mathbb{P}\left(t_{1}^{-} \leq B\right)+o(\varepsilon)$.

To estimate $\mathbb{P}\left(t_{1}^{-} \leq B\right)$, let $\left(D_{i}\right)$ denote the sequence of departures times and $N$ the number of customers served during the busy period of length $B$, then 
Equation (2) gives the identity

$$
\begin{aligned}
\mathbb{P}\left(t_{1}^{-} \leq B\right) & =\mathbb{E}\left(\sum_{i=1}^{N} \frac{\varepsilon p^{-}\left(X\left(D_{i}\right)\right)}{\mu} \prod_{j=1}^{i-1}\left(1-\frac{\varepsilon p^{-}\left(X\left(D_{j}\right)\right)}{\mu}\right)\right) \\
& =\frac{\varepsilon}{\mu} \mathbb{E}\left(\sum_{i=1}^{N} p^{-}\left(X\left(D_{i}\right)\right)\right)+o(\varepsilon)=\frac{\varepsilon}{\mu} \mathbb{E}(N) \mathbb{E}\left[p^{-}\left(X\left(D_{1}\right)\right)\right]+o(\varepsilon)
\end{aligned}
$$

by stationarity of $(X(t))$ and Wald's Formula with $\mathbb{E}(N)=1 /(1-\rho)$. Similarly,

$$
\begin{aligned}
\mathbb{E}\left(\left(B-t_{1}^{-}\right) \mathbb{1}_{\left\{t_{1}^{-} \leq B\right\}}\right) & =\frac{\varepsilon}{\mu} \mathbb{E}\left(\sum_{i=1}^{N} p^{-}\left(X\left(D_{i}\right)\right)\left(B-D_{i}\right)\right)+o(\varepsilon) \\
& =\varepsilon \frac{\mathbb{E}\left[p^{-}\left(X\left(D_{1}\right)\right)\right]}{\mu}(\mathbb{E}(N B)-E(D))
\end{aligned}
$$

where $D=\sum_{i=1}^{N} D_{i}$ is the sum of the departures in the busy period of the SQueue. Using the fact that $\mathbb{E}(D)=\mu^{2} /(\mu-\lambda)^{3}, \mathbb{E}(N B)=(1+\rho) /\left(\mu(1-\rho)^{3}\right)$ and $\mathbb{E}(\mathcal{A})=\mu /(\mu-\lambda)^{2}$, the result is proved.

Combining Lemmas 5 and 6 yields the first order term indicated in Theorem 1.

\section{A.2 Second order term}

To compute the second order term in the power series expansion in $\varepsilon$ of $\mathbb{E}\left(\Delta^{\varepsilon}\right)$, three cases have to be considered:

$-t_{1}^{+} \leq \widetilde{B}^{\varepsilon}$ or $t_{2}^{+} \leq \widetilde{B}^{\varepsilon}$ : one or two additional departures occur in a busy period;

$-t_{1}^{-} \leq \widetilde{B}^{\varepsilon}$ or $t_{2}^{-} \leq \widetilde{B}^{\varepsilon}$ : one or two departures are canceled;

$-t_{1}^{+} \leq \widetilde{B}^{\varepsilon}$ and $t_{1}^{-} \leq \widetilde{B}^{\varepsilon}$ : one additional departure takes place and another one is canceled.

It is not difficult to show that any event involving a third jump yields a term of the order $\varepsilon^{3}$ in the expansion of the mean bit rate. Due to the space constraints, a part of the expansion is proved. The complete proofs of the expansion can be found in Antunes et al. (12).

In the case that two additional departures occur during $\widetilde{B}^{\varepsilon}$, the difference between the areas of the busy periods due to the first additional jump is given by the two first terms on the right hand side of the following equation

$$
\begin{array}{r}
\mathbb{E}\left(\Delta^{\varepsilon} \mathbb{1}_{\left\{t_{1}^{+} \leq B, t_{2}^{+} \leq t_{1}^{+}+B_{L\left(t_{1}^{+}\right)-1}\right\}}\right)=\mathbb{E}\left(\left(B-t_{1}^{+}\right) \mathbb{1}_{\left\{t_{1}^{+} \leq B, t_{2}^{+} \leq t_{1}^{+}+B_{L\left(t_{1}^{+}\right)-1}\right\}}\right) \\
+\mathbb{E}\left(\overline{\mathcal{A}}_{B_{1}}\right) \mathbb{P}\left(t_{1}^{+} \leq B, t_{2}^{+} \leq t_{1}^{+}+B_{L\left(t_{1}^{+}\right)-1}\right)+\mathbb{E}\left(B_{L\left(t_{2}^{+}\right)-1} \mathbb{1}_{\left\{t_{1}^{+} \leq B, t_{2}^{+}<t_{1}^{+}+B_{L\left(t_{1}^{+}\right)-1}\right\}}\right) \\
+\mathbb{E}\left(\overline{\mathcal{A}}_{B_{1}^{\prime}}\right) \mathbb{P}\left(t_{1}^{+} \leq B, t_{2}^{+}<t_{1}^{+}+B_{L\left(t_{1}^{+}\right)-1}\right)+o\left(\varepsilon^{2}\right), \quad(\mathrm{A} .2)
\end{array}
$$


which follows by the same arguments stated for only one additional departure.

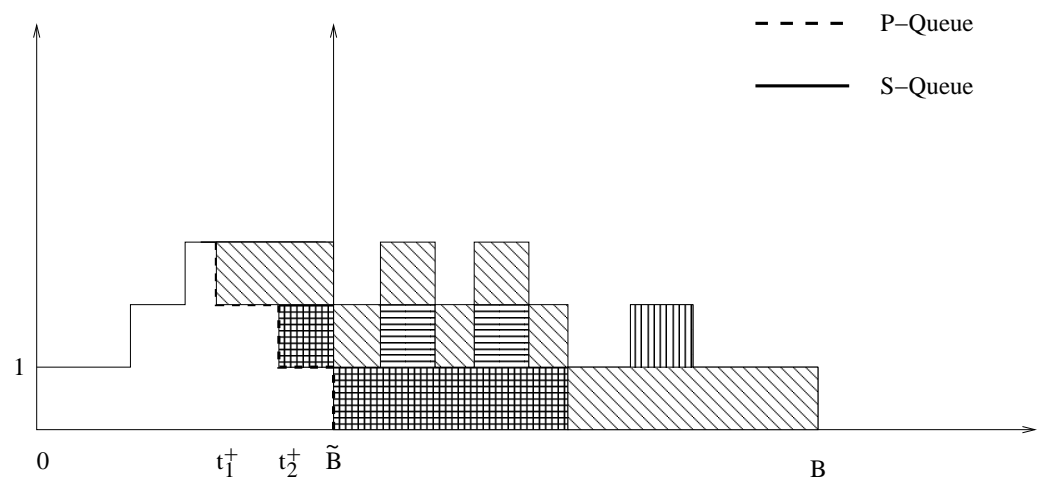

Fig. A.2. Two additional departures

Due to the second additional jump, the difference $\Delta^{\varepsilon}$ increases by the sum of two disjoint areas. The first one is given by $B_{L\left(t_{2}^{+}\right)-1}$ and represents the distance from the second additional jump until the first time the S-Queue with less than one customer is empty. Note that by conditioning on the event $\left\{t_{1}^{+} \leq B, t_{2}^{+} \leq t_{1}^{+}+B_{L\left(t_{1}^{+}\right)-1}\right\}$, at the stopping time $\widetilde{B}^{\varepsilon}$, a new busy period $B_{1}^{\prime}$ starts with the same distribution as $B$. Thus, the second area is given by the area of sub-busy periods in $\mid w B_{1}$ (periods where $t \rightarrow L(t)$ is $>1$ ) in $B_{1}^{\prime}$, hence

$$
\begin{aligned}
\mathbb{E}\left(\Delta^{\varepsilon} \mathbb{1}_{\mathcal{E}_{+}}\right)= & \mathbb{E}\left(\Delta^{\varepsilon} \mathbb{1}_{\left\{t_{1}^{+} \leq B, t_{2}^{+} \geq t_{1}^{+}+B_{L\left(t_{1}^{+}\right)-1}, t_{1}^{-} \geq t_{1}^{+}+B_{L\left(t_{1}^{+}\right)-1}\right\}}\right) \\
& +E\left(\Delta^{\varepsilon} \mathbb{1}_{\left\{t_{1}^{+} \leq B, t_{2}^{+} \leq t_{1}^{+}+B_{L\left(t_{1}^{+}\right)-1}\right\}}\right)+o\left(\varepsilon^{2}\right) \\
=\mathbb{E}\left(\left(B-t_{1}^{+}\right) \mathbb{1}_{\left\{t_{1}^{+} \leq B\right\}}\right)+ & \mathbb{E}\left(B_{L\left(t_{2}^{+}\right)-1} \mathbb{1}_{\left\{t_{1}^{+} \leq B, t_{2}^{+}<t_{1}^{+}+B_{L\left(t_{1}^{+}\right)-1}\right\}}\right)+\mathbb{E}\left(\overline{\mathcal{A}}_{B_{1}}\right) \mathbb{P}\left(t_{1}^{+}<B\right) \\
+ & \mathbb{E}\left(\overline{\mathcal{A}}_{B_{1}^{\prime}}\right) \mathbb{P}\left(t_{1}^{+} \leq B, t_{2}^{+}<t_{1}^{+}+B_{L\left(t_{1}^{+}\right)-1}\right)+K(\varepsilon)+o\left(\varepsilon^{2}\right) . \quad \text { (A.3) }
\end{aligned}
$$

where $K(\varepsilon)$ is a term which is not expressed here for sake of simplicity. The following proposition gives the expansion of some of the terms of Equation (A.3). The other expansions are done much in the same way (with various complications).

Proposition 7 The following expansions hold

$$
\begin{aligned}
& \mathbb{P}\left(t_{1}^{+} \leq B\right)= \\
& \varepsilon \frac{\mathbb{E}\left[p^{+}(X(0))\right]}{\mu-\lambda}-\varepsilon^{2} \mathbb{E}\left(\int_{0}^{B}(B-v) \mathbb{E}\left[p^{+}(X(0)) p^{+}(X(v))\right] d v\right)+o\left(\varepsilon^{2}\right), \\
& \begin{array}{r}
\mathbb{P}\left(t_{1}^{+}<B, t_{2}^{+} \leq t_{1}^{+}+B_{L\left(t_{1}^{+}\right)-1}\right) \\
=\varepsilon^{2} \rho \mathbb{E}\left(\int_{0}^{B}(B-v) \mathbb{E}\left[p^{+}(X(0)) p^{+}(X(v))\right] d v\right)+o\left(\varepsilon^{2}\right) .
\end{array}
\end{aligned}
$$


Proof. Since $\mathbb{P}\left(t_{1}^{+} \leq B\right)=\mathbb{E}\left(1-\exp \left(-\varepsilon \int_{0}^{B} p^{+}(X(s)) d s\right)\right)$, the expansion in power series of $\varepsilon$ has the first term $\mathbb{E}\left(p^{+}(X(0))\right) /(\mu-\lambda)$ and second term

$$
\begin{aligned}
\frac{1}{2} \mathbb{E}\left(\left(\int_{0}^{B} p^{+}(X(s)) d s\right)^{2}\right) & =\mathbb{E}\left(\int_{0 \leq u \leq v \leq B} \mathbb{E}\left(p^{+}(X(0)) p^{+}(X(v-u))\right) d u d v\right) \\
& =\mathbb{E}\left(\int_{0}^{B}(B-v) \mathbb{E}\left[p^{+}(X(0)) p^{+}(X(v))\right] d v\right)
\end{aligned}
$$

by stationarity of the process $(X(t))$. The first expansion is proved.

The event $\left\{t_{1}^{+} \leq B, t_{2}^{+}<t_{1}^{+}+B_{L\left(t_{1}^{+}\right)-1}\right\}$ occurs only when $t_{1}^{+}$and $t_{2}^{+}$are in a sub-busy period $\left[s_{i-1}+E_{i}, s_{i}\right]$, for some $i \in\{1, \ldots, H\}$, (a period where $L$ is always $>1)$. The variables $E_{i}$ are i.i.d. exponential with parameter $\lambda$, $B_{1}^{i}=s_{i}-s_{i-1}-E_{i-1}$ has the same distribution as $B$ and $H$ is geometrically distributed with parameter $\lambda /(\lambda+\mu)$. The probability that the first two additional jumps are in the $i$-th sub-busy period, is

$$
\begin{aligned}
\mathbb{E}\left(\int_{s_{i-1}+E_{i}}^{s_{i}} \varepsilon p^{+}(X(u)) e^{-\varepsilon \int_{0}^{u} p^{+}(X(s)) d s}\left(1-e^{-\varepsilon \int_{u}^{s_{i}} p^{+}(X(s)) d s}\right) d u\right) \\
=\varepsilon^{2} \mathbb{E}\left(\int_{s_{i-1}+E_{i}}^{s_{i}} p^{+}(X(u)) \int_{u}^{s_{i}} p^{+}(X(s)) d s d u\right)+o\left(\varepsilon^{2}\right) \\
=\varepsilon^{2} \mathbb{E}\left(\int_{0 \leq u \leq v \leq B} \mathbb{E}_{\nu}\left(p^{+}(X(0)) p^{+}(X(v-u))\right) d u\right)+o\left(\varepsilon^{2}\right),
\end{aligned}
$$

which gives the second expansion.

The complete expansion is now detailed.

Proposition 8 The coefficients of $\varepsilon^{2}$ in the expansion of $\mathbb{E}\left(\Delta^{\varepsilon}\right)$ are given by

$$
\begin{aligned}
a_{+}=-\frac{\rho}{\mu(1-\rho)} \mathbb{E}\left(\int_{0}^{B}(B-v) \mathbb{E}\left[p^{+}(X(0)) p^{+}(X(v))\right] d v\right) \\
-\frac{1-\rho}{2} \mathbb{E}\left(\int_{0}^{B}(B-u)^{2} \mathbb{E}\left[p^{+}(X(0)) p^{+}(X(u))\right] d u\right), \\
a_{-}=-\frac{1}{\mu^{3}(1-\rho)} \mathbb{E}\left(\sum_{1 \leq i<j \leq N} p^{-}\left(X\left(D_{i}\right)\right) p^{-}\left(X\left(D_{j}\right)\right)\right) \\
-\frac{1}{\mu^{2}} \mathbb{E}\left(\sum_{i=1}^{N} \sum_{j=1}^{N^{\prime}} p^{-}\left(X\left(D_{i}\right)\right) p^{-}\left(X\left(B+D_{j}^{\prime}\right)\right)\left(B_{1}-D_{j}^{\prime}+\frac{\mu}{(\mu-\lambda)^{2}}\right)\right),
\end{aligned}
$$




$$
\begin{aligned}
a_{ \pm} & =\frac{1}{\mu} \mathbb{E}\left(\sum_{i=1}^{N} \int_{0}^{B} p^{-}\left(X\left(D_{i}\right)\right) p^{+}(X(s)) d s\left(\frac{\mu}{(\mu-\lambda)^{2}}+B-D_{i}\right)\right) \\
& +\frac{1}{\mu} \mathbb{E}\left(\sum_{i=1}^{N} \int_{0}^{B_{1}} p^{-}\left(X\left(D_{i}\right)\right) p^{+}(X(B+s))\left(B_{1}-s+\frac{\lambda}{(\mu-\lambda)^{2}}\right) d s\right) \\
- & \frac{1}{\mu} \mathbb{E}\left(\sum_{i=1}^{H} \sum_{k=1}^{N_{i}} \int_{0}^{A_{i}} p^{+}(X(u)) p^{-}\left(X\left(D_{i}^{k}\right)\right)\left(\frac{\lambda}{(\mu-\lambda)^{2}}+A_{i}-u\right) d u\right)
\end{aligned}
$$

where $H$ is geometric distributed with parameter $\lambda /(\mu+\lambda),\left(N_{i}, D_{1}^{i}, \ldots D_{N_{i}}^{i}\right)$ denotes respectively the number of departures and the departures times in a busy period $B_{1}^{i}$, and $A_{i}=B_{1}^{i}+E_{0}+\sum_{k=i+1}^{H}\left(E_{k}+B_{1}^{k}\right)$ where $\left(E_{i}\right)$ are i.i.d is exponentially distributed with parameter $\mu+\lambda$ and $\left(B_{1}^{i}\right)$ are i.i.d with the same distribution as $B$.

\section{References}

[1] L. Massoulié, J. Roberts, Bandwidth sharing: Objectives and algorithms, in: INFOCOM '99. Eighteenth Annual Joint Conference of the IEEE Computer and Communications Societies, 1999, pp. 1395-1403.

[2] F. Delcoigne, A. Proutière, G. Régnié, Modeling integration of streaming and data traffic, in: ITC specialist seminar on IP traffic, Würzburg, Germany, 2002.

[3] T. Bonald, A. Proutière, On performance bounds for the integration of elastic and adaptive streaming traffic, in: ACM Sigmetrics/Performance, 2004.

[4] N. Antunes, C. Fricker, F. Guillemin, P. Robert, Perturbation analysis of a variable $M / M / 1$ queue: a probabilistic approach, submitted for publication (2005).

[5] R. Núñez-Queija, O. Boxma, Analysis of a multi-server queueing model of ABR, J. Appl. Math. Stoch. An. 11 (1998) 339-354.

[6] R. Núñez-Queija, Sojourn times in non-homogeneous QBD processes with processor sharing, Stoch. Mod. (2001) 61-92.

[7] R. Núñez-Queija, Sojourn times in a processor sharing queue with service interruptions, Queueing Systems 34 (2000) 351-386.

[8] R. Núñez Queija, E. Altman, K. Avrachenkov, Perturbation analysis for denumerable markov chains with application to queueing models, Advances in Applied Probability 36 (3) (2004) 839-853.

[9] O. Boxma, I. Kurkova, The $M / G / 1$ queue with two service speeds, Advances in Applied Probability 33 (2) (2002) 520-540.

[10] C. Fricker, F. Guillemin, P. Robert, Perturbation analysis of an M/M/1 queue in a diffusion random environment, preprint (Jan. 2004).

[11] J. Grandell, Point processes and random measures, Advances in Applied Probability 9 (1977) 502-526. 
[12] N. Antunes, C. Fricker, F. Guillemin, P. Robert, Perturbation analysis of the area swept under the queue length process of a variable $M / M / 1$ queue, URL: http://www-rocq.inria.fr/ ${ }^{\sim}$ robert/src/papers/2005-1full.pdf (January 2005).

[13] J. W. Cohen, The single server queue, 2nd Edition, North-Holland, Amsterdam, 1982. 